\title{
Loop formulation of supersymmetric Yang-Mills quantum mechanics
}

\author{
Kyle Steinhauer and Urs Wenger \\ Albert Einstein Center for Fundamental Physics, \\ Institute for Theoretical Physics, University of Bern, \\ Sidlerstrasse 5, CH-3012 Bern, Switzerland \\ E-mail: steinhauer@itp.unibe.ch, wenger@itp.unibe.ch
}

ABSTRACT: We derive the fermion loop formulation of $\mathcal{N}=4$ supersymmetric $\operatorname{SU}(N)$ Yang-Mills quantum mechanics on the lattice. The loop formulation naturally separates the contributions to the partition function into its bosonic and fermionic parts with fixed fermion number and provides a way to control potential fermion sign problems arising in numerical simulations of the theory. Furthermore, we present a reduced fermion matrix determinant which allows the projection into the canonical sectors of the theory and hence constitutes an alternative approach to simulate the theory on the lattice.

Keywords: Field Theories in Lower Dimensions, Lattice Gauge Field Theories, M(atrix) Theories, Black Holes in String Theory

ARXIV EPRINT: 1410.0235 


\section{Contents}

1 Introduction 1

2 Lattice regularisation 3

3 Fermion matrix reduction and canonical formulation 4

4 Fermion loop formulation $\quad 6$

5 Fermion sectors and transfer matrices $\quad \mathbf{8}$

5.1 Sector $n_{f}=0 \quad 9$

$\begin{array}{lll}5.2 \text { Sector } n_{f}=n_{f}^{\max } & 10\end{array}$

$\begin{array}{lll}5.3 \text { Sector } n_{f}=1 & 10\end{array}$

5.4 Sector $n_{f}=n_{f}^{\max }-1 \quad 11$

$\begin{array}{ll}5.5 \text { Sector with generic } n_{f} & 12\end{array}$

$\begin{array}{lll}5.6 & \text { Remarks } & 13\end{array}$

6 Conclusions and outlook $\quad 14$

A Determinations of canonical determinants $\quad \mathbf{1 5}$

$\begin{array}{lll}\text { A.1 Coefficients from recursion relations } & 15\end{array}$

$\begin{array}{lll}\text { A.2 Coefficients in terms of traces } & 16\end{array}$

$\begin{array}{lll}\text { A.3 Coefficients in terms of minors } & 16\end{array}$

$\begin{array}{ll}\text { B Equivalence of canonical determinants } & 17\end{array}$

\section{Introduction}

The conjectured holographic duality between supersymmetric Yang-Mills quantum mechanics and the theory of D0 branes of type IIa string theory in the large- $N$ limit in principle allows to probe the physics of certain supergravity black holes by lattice Monte Carlo simulations. In particular, $\mathcal{N}=16$ supersymmetric Yang-Mills (SYM) quantum mechanics (QM) stemming from the dimensional reduction of $\mathcal{N}=1 \mathrm{SYM}$ in $d=10$ dimensions is supposed to describe the dynamics of D0 branes which are the degrees of freedom of the underlying M-theory [1]. The connection to so-called black p-branes allows to study the thermodynamics of black holes through the corresponding strongly coupled gauge theory. We refer the reader to the review article [2] for further details. Here we report on our work in this direction on an analogue, but simpler theory, namely $\mathcal{N}=4$ SYM QM with generic gauge group $\mathrm{SU}(N)$. The model stems from dimensionally reducing $\mathcal{N}=1 \mathrm{SYM}$ in $d=4$ dimensions, but is expected to share many qualitative features with the 16 supercharge 
model. The aim of this paper is to construct the fermion loop formulation of the strongly coupled gauge theory regularised on the lattice, so as to make it susceptible to numerical simulations.

There have already been a number of nonperturbative investigations of SYM QM using numerical techniques. In [3-7] the Hamiltonian formulation was employed together with the cut Fock space method. This approach also allowed analytic solutions, at least for $d=2$ dimensional SYM QM [8-12]. On the other hand, in [13] the Wilson lattice discretization was constructed and the $d=4$ SYM QM was simulated in the quenched approximation $[13,14]$. Further discretizations were proposed and investigated by Monte Carlo simulations in [15-17], and it was also shown that the (naive) Wilson discretization does not require any fine tuning to reach the correct continuum limit. A different nonlattice approach has been followed by [18-21] which used a momentum cutoff regularization while completely fixing the gauge.

Our motivation to study the loop formulation of this model is threefold. Apart from the motivation given by the interesting physics related to the thermodynamics of black holes and the possibility to test the gauge/gravity duality outlined above, the loop formulation provides a new approach to simulate fermions on the lattice [22]. In contrast to standard approaches the fermion loop formulation allows for local fermion algorithms [23], i.e., local updates of the fermionic degrees of freedom. The simulation algorithm applicable to the loop formulation works for massless fermions and appears not to suffer from critical slowing down $[23,24]$. This is of particular importance in the context of supersymmetric field theories with spontaneously broken supersymmetry, since in such cases one has to deal with a massless fermionic mode, the Goldstino fermion. The third motivation finally stems from the fact that the fermion loop formulation offers the potential to control the fermion sign problem. Again, this is of particular significance in theories with spontaneously broken supersymmery where the partition function for periodic boundary conditions, and hence the fermion determinant (or Pfaffian), averages to zero, since it represents the vanishing Witten index [22, 25-27]. The possibility to control the fermion sign then follows from the fact that in the loop formulation the fermionic contribution to the partition function decomposes into contributions from fixed fermion number sectors, each of which has a definite sign depending only on the specific choice of the fermionic boundary conditions.

The paper is organised as follows. In section 2 we discuss the $d=4$ dimensional SYM QM in the continuum and describe the lattice regularisation using the Wilson derivative. In section 3 we derive a reduction formula for the determinant of the fermion matrix which separates the dependence of the bosonic degrees of freedom from the chemical potential and which then allows the straightforward discussion of the canonical sectors of the theory. In section 4 the fermion loop formulation is introduced and in section 5 we discuss the various fermion sectors emerging from the transfer matrices in the loop formulation. We close the main part of the paper with our conclusions and an outlook in section 6. Finally, in appendix A we review various ways how to determine the canonical determinants from the reduced fermion matrix and prove in appendix $\mathrm{B}$ the algebraic equivalence between the reduced fermion matrix approach and the fermion loop formulation. 


\section{Lattice regularisation}

We start from $\mathcal{N}=1 \mathrm{SYM}$ in $d=4$ dimensions with gauge group $\mathrm{SU}(N)$ and dimensionally reduce the theory by compactifying the three spatial dimensions. While the temporal component $A(t)$ of the 4-dimensional gauge field remains unchanged, the three spatial components become bosonic fields $X_{i}(t), i=1,2,3$. The action of the dimensionally reduced theory then reads

$$
S=\frac{1}{g^{2}} \int_{0}^{\beta} d t \operatorname{Tr}\left\{\left(D_{t} X_{i}\right)^{2}-\frac{1}{2}\left[X_{i}, X_{j}\right]^{2}+\bar{\psi} D_{t} \psi-\bar{\psi} \sigma_{i}\left[X_{i}, \psi\right]\right\}
$$

where the anticommuting fermion fields $\bar{\psi}(t), \psi(t)$ are complex 2-component spinors, $\sigma_{i}$ are the three Pauli matrices and $D_{t}=\partial_{t}-i[A(t), \cdot]$ denotes the covariant derivative. All fields in the theory are in the adjoint representation of $\mathrm{SU}(N)$ and the theory possesses a $\mathcal{N}=4$ supersymmetry.

Note that the analogue reduction from $\mathcal{N}=1 \mathrm{SYM}$ in $d=10$ dimensions yields a very similar action with the only change that there are 9 bosonic fields $X_{i}(t), i=1, \ldots, 9$ corresponding to the 9 compactified gauge degrees of freedom, the $\sigma_{i}$ 's are the $\mathrm{SO}(9) \gamma$ matrices and the fermionic Grassmann variables are Majorana, i.e., can be taken to be real. The dimensionally reduced theory then corresponds to $\mathcal{N}=16 \mathrm{SYM}$ QM.

Let us now describe the lattice regularised version of the $\mathcal{N}=4$ SYM QM where the Euclidean time extent is discretised by $L_{t}$ points. The bosonic part of the action is then given by

$$
S_{B}=\frac{1}{g^{2}} \sum_{t=0}^{L_{t}-1} \operatorname{Tr}\left\{\hat{D}_{t} X_{i}(t) \hat{D}_{t} X_{i}(t)-\frac{1}{2}\left[X_{i}(t), X_{j}(t)\right]^{2}\right\}
$$

where the gauge field is replaced by the gauge link $U(t)$ living in the gauge group $\mathrm{SU}(N)$ and the covariant lattice derivative is explicitly given by $\hat{D}_{t} X_{i}(t)=U(t) X_{i}(t+1) U^{\dagger}(t)-X_{i}(t)$. For the regularisation of the fermionic part we use the Wilson discretisation to get rid of the fermion doublers. Note that in $d=1$ dimensions adding a Wilson term with Wilson parameter $r= \pm 1$ to the symmetric derivative yields either a forward or backward derivative,

$$
\partial^{\mathcal{W}}=\frac{1}{2}\left(\nabla^{+}+\nabla^{-}\right) \pm \frac{1}{2} \nabla^{+} \nabla^{-}=\nabla^{ \pm} .
$$

Hence, the discretised fermion action reads

$$
S_{F}=\frac{1}{g^{2}} \sum_{t=0}^{L_{t}-1} \operatorname{Tr}\left\{\bar{\psi}(t) \hat{D}_{t} \psi(t)-\bar{\psi}(t) \sigma_{i}\left[X_{i}(t), \psi(t)\right]\right\}
$$

where $\hat{D}_{t}$ is simply the covariant derviative defined above. Note that the Wilson term breaks the time reversal and hence also the charge conjugation symmetry. However, the symmetries are restored in the continuum limit together with the full supersymmetries without any fine tuning since any further symmetry breaking terms are prohibited by the gauge symmetry [15].

For our further discussion of the fermionic part of the theory, it is convenient to work in uniform gauge $U(t)=U$, although it is not necessary for the derivation of the reduced 
fermion matrix in the next section. In addition, we also include a finite chemical potential term $e^{\mu}$ in the forward fermion derivative [28] in order to facilitate our discussion of the canonical fermion sectors in the next section. To be specific, the fermion action then reads

$$
S_{F}=\frac{1}{2 g^{2}} \sum_{t=0}^{L_{t}-1}\left[-\bar{\psi}_{\alpha}^{a}(t) W_{\alpha \beta}^{a b} e^{\mu} \psi_{\beta}^{b}(t+1)+\bar{\psi}_{\alpha}^{a}(t) \Phi_{\alpha \beta}^{a b}(t) \psi_{\beta}^{b}(t)\right]
$$

where the gauge part of the hopping term connecting the nearest neighbour Grassmann fields $\bar{\psi}_{\alpha}^{a}(t)$ and $\psi_{\beta}^{b}(t+1)$ is given by

$$
W_{\alpha \beta}^{a b}=2 \delta_{\alpha \beta} \cdot \operatorname{Tr}\left\{T^{a} U T^{b} U^{\dagger}\right\}
$$

and is independent of $t$. Here, $T^{a}$ are the generators of the $\mathrm{SU}(N)$ algebra and are normalised such that det $W=1$. The Yukawa interaction between the fermionic and bosonic fields is described by a $2\left(N^{2}-1\right) \times 2\left(N^{2}-1\right)$ matrix

$$
\Phi_{\alpha \beta}^{a b}(t)=\left(\sigma_{0}\right)_{\alpha \beta} \cdot \delta^{a b}-2\left(\sigma_{i}\right)_{\alpha \beta} \cdot \operatorname{Tr}\left\{T^{a}\left[X_{i}(t), T^{b}\right]\right\}
$$

and the fermion action can be compactly written in terms of the fermion Dirac matrix $\mathcal{D}_{p, a}$, i.e.,

$$
S_{F}=\frac{1}{2 g^{2}} \bar{\psi} \mathcal{D}_{p, a}\left[U, X_{i} ; \mu\right] \psi .
$$

where the subscripts $p, a$ specify periodic or antiperiodic temporal boundary conditions for the fermions in time, $\psi\left(L_{t}\right)= \pm \psi(0)$, respectively.

Eventually, the grand canonical partition function reads

$$
Z=\int \mathcal{D} U \mathcal{D} X_{i} e^{-S_{B}\left[U, X_{i}\right]} \operatorname{det} \mathcal{D}_{p, a}\left[U, X_{i} ; \mu\right]
$$

where the determinant of the fermion Dirac matrix is the result from integrating out the fermionic degrees of freedom $\bar{\psi}$ and $\psi$.

\section{Fermion matrix reduction and canonical formulation}

In $d=1$ dimensions the fermion matrix is particularly simple and takes a cyclic block bidiagonal form,

$$
\mathcal{D}_{p, a}=\left(\begin{array}{ccccc}
\Phi(0) & -W e^{\mu} & & & \\
& \Phi(1) & -W e^{\mu} & & \\
& \Phi(2) & \ddots & \\
& & & \ddots & -W e^{\mu} \\
& & & & \Phi\left(L_{t}-1\right)
\end{array}\right) .
$$

Subsequently, determinant reduction techniques based on Schur complements similar to the ones described in [29] can be applied. As a consequence the grand canonical determinant for the reduced fermion matrix yields

$$
\operatorname{det} \mathcal{D}_{p, a}\left[U, X_{i} ; \mu\right]=\operatorname{det}\left[\mathcal{T} \mp e^{+\mu L_{t}}\right]
$$


where $\mathcal{T}$ is the simple matrix product

$$
\mathcal{T}=\prod_{t=0}^{L_{t}-1}(\Phi(t) W)
$$

For given background fields $U$ and $X_{i}(t)$ the formula allows to calculate the determinant for any value of the chemical potential $\mu$ by simply diagonalising $\mathcal{T}$ and evaluating the characteristic polynomial of order $2\left(N^{2}-1\right)$ in $e^{\mu L_{t}}$. The coefficients of the polynomial are then just the fermion contributions to the grand canonical partition functions [29],

$$
\operatorname{det} \mathcal{D}_{p, a}\left[U, X_{i} ; \mu\right]=\sum_{n_{f}=0}^{2\left(N^{2}-1\right)}\left(\mp e^{\mu L_{t}}\right)^{n_{f}} \operatorname{det} \mathcal{D}_{n_{f}}\left[U, X_{i}\right]
$$

which is the conventional fugacity expansion. Note that the computational effort to evaluate eq. (3.2) grows only linearly with the temporal extent of the lattice (through the number of multiplications in the product), for example as one takes the continuum limit $L_{t} \rightarrow \infty$. One can also work in temporal gauge in which all gauge links are transformed to unity except one denoted by $\widetilde{W}$, e.g., the one connecting time slice $t=L_{t}-1$ and $t=0$. The relation to the uniform gauge is then $\widetilde{W}=W^{L_{t}}$ and the product becomes $\prod_{t=0}^{L_{t}-1} \Phi(t) \cdot \widetilde{W}$. Finally we note that for ordinary supersymmetric quantum mechanics the expression for $\mathcal{T}$ reduces to the result given in [30].

Next we turn to the explicit evaluation of the canonical determinants. Denoting the eigenvalues of $\mathcal{T}$ in eq. (3.3) by $\tau_{j}, j=1, \ldots, 2\left(N^{2}-1\right)$ we can express the determinants directly in terms of these by comparing the coefficients of the characteristic polynomial

$$
\operatorname{det} \mathcal{D}_{p, a}\left[U, X_{i} ; \mu\right]=\prod_{j=1}^{2\left(N^{2}-1\right)}\left(\tau_{j} \mp e^{\mu L_{t}}\right)
$$

with eq. (3.4). The canonical determinant in the sector with $n_{f}=2\left(N^{2}-1\right) \equiv n_{f}^{\max }$ fermions is trivial,

$$
\operatorname{det} \mathcal{D}_{n_{f}^{\max }}\left[U, X_{i}\right]=1,
$$

which simply reflects the fact that the sector with maximally saturated fermion number is quenched. For the sector with $n_{f}=0$ we obtain

$$
\operatorname{det} \mathcal{D}_{n_{f}=0}\left[U, X_{i}\right]=\prod_{j=1}^{2\left(N^{2}-1\right)} \tau_{j}=\operatorname{det}\left[\prod_{t=0}^{L_{t}-1}(\Phi(t) W)\right]=\operatorname{det}\left[\prod_{t=0}^{L_{t}-1} \Phi(t)\right]
$$

where we made use of the fact that $\operatorname{det} W=1$. The formula shows that the fermion contribution in the $n_{f}=0$ sector is nontrivial, even though it is independent of the gauge link $U$.

The sectors with $n_{f}=1$ and $n_{f}=n_{f}^{\max }-1$ fermions are similarly simple,

$$
\operatorname{det} \mathcal{D}_{n_{f}=1}=\sum_{j=1}^{2\left(N^{2}-1\right)} \prod_{k \neq j} \tau_{k}
$$




$$
\operatorname{det} \mathcal{D}_{n_{f}=n_{f}^{\max }-1}=\sum_{j=1}^{2\left(N^{2}-1\right)} \tau_{j}=\operatorname{Tr}(\mathcal{T}) .
$$

The generic formula for the canonical determinants in terms of the eigenvalues can be expressed by the elementary symmetric functions $S_{k}$ of the $n_{f}^{\max }$ eigenvalues $\tau_{1}, \ldots, \tau_{n_{f}^{\max }}$ with $k \leq n_{f}^{\max }$. The $k^{\text {th }}$ elementary symmetric function is defined as

$$
S_{k}(\mathcal{T}) \equiv S_{k}\left(\tau_{1}, \ldots, \tau_{n_{f}^{\max }}\right)=\sum_{1 \leq i_{1}<\cdots<i_{k} \leq n_{f}^{\max }} \prod_{j=1}^{k} \tau_{i_{j}},
$$

where the sum has $\left(\begin{array}{c}n_{f}^{\max } \\ k\end{array}\right)$ summands, and the canonical determinant in the sector with $n_{f}$ fermions eventually reads

$$
\operatorname{det} \mathcal{D}_{n_{f}}=S_{n_{f}^{\max }-n_{f}}(\mathcal{T}) \text {. }
$$

Of course the coefficients of the characteristic polynomial can be obtained in many other ways. In appendix A we present several alternative methods how to calculate the canonical determinants directly from the matrix $\mathcal{T}$. One method makes use of the traces of powers of $\mathcal{T}$ while the other employs the minors of $\mathcal{T}$. The latter turns out to be closely related to the transfer matrices emerging from the fermion loop formulation discussed in the next section.

\section{Fermion loop formulation}

In the fermion loop formulation the decomposition into the various fermion sectors are recovered in a completely different and independent way. The formulation is based on the exact hopping expansion of the fermion Boltzmann factor involving the action in eq. (2.5). Since the overall prefactor $1 / 2 g^{2}$ only contributes a trivial factor we suppress it in the following. We apply the expansion not only to the hopping term, but in fact to all terms in the fermion action including the Yukawa term. The expansion is exact because it naturally truncates after the first two terms due to the nilpotency of the Grassmann variables. Such an expansion is most conveniently expressed by

$$
e^{x}=1+x=\sum_{m=0}^{1} x^{m},
$$

i.e., in terms of occupation numbers $m$. Applying this equation to each term in the fermion action eq. (2.5) characterised by the colour indices $a, b$, the Dirac algebra indices $\alpha, \beta$ and the time coordinate $t$, the expansion of the fermion Boltzmann factor yields

$$
\begin{array}{r}
\exp \left(-S_{F}\right)=\prod_{t, a, b, \alpha, \beta}\left[\sum_{m_{\alpha \beta}^{a b}(t)=0}^{1}\left(-\Phi_{\alpha \beta}^{a b}(t) \bar{\psi}_{\alpha}^{a}(t) \psi_{\beta}^{b}(t)\right)^{m_{\alpha \beta}^{a b}(t)}\right] \\
\times \prod_{t, a, \alpha}\left[\sum_{h_{\alpha \beta}^{a b}(t)=0}^{1}\left(\bar{\psi}_{\alpha}^{a}(t) W_{\alpha \beta}^{a b} \psi_{\beta}^{b}(t+1)\right)^{h_{\alpha \beta}^{a b}(t)}\right],
\end{array}
$$




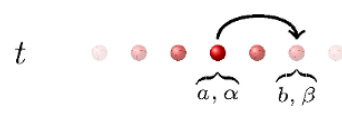

weight: $\Phi_{\alpha \beta}^{a b}(t)$

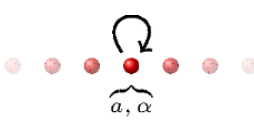

weight: $\Phi_{\alpha \alpha}^{a a}(t)$

Figure 1. Graphical representation of the Yukawa interaction between the fermionic degree of freedom characterised by $(a, \alpha)$ on time slice $t$ with the one characterised by $(b, \beta)$ on the same time slice and $(a, \alpha)$ with itself (monomer term). The contributions of the interactions (weights) after the Grassmann integrations are also given.

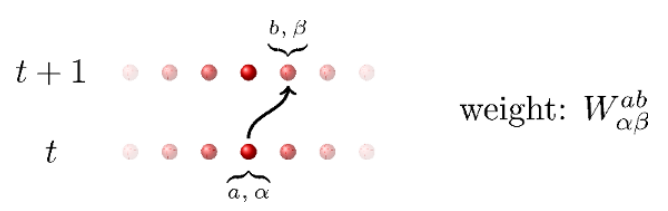

Figure 2. Graphical representation of a gauged temporal hop connecting the fermionic degree of freedom characterised by $(a, \alpha)$ on time slice $t$ with the one characterised by $(b, \beta)$ on time slice $t+1$. The contribution of the hop (weight) after the Grassmann integrations is also given.

Here, the terms in the first product follow from the Yukawa interaction while the terms in the second product stem from the hopping terms in which we have put $\mu=0$ to simplify the discussion. Note that one has a separate expansion for every combination of indices $t, a, b, \alpha, \beta$ which stops after the first two terms due to the Grassmannian character of the fermionic degrees of freedom. The two terms in each expansion are characterised by the occupation numbers $h_{\alpha \beta}^{a b}(t)$ and $m_{\alpha \beta}^{a b}(t)$ taking the values 0 or 1 . The Grassmann integration over the fermion fields requires that every pair $\bar{\psi}_{\alpha}^{a}(t) \psi_{\alpha}^{a}(t)$ needs to be saturated by the integration measure in order to give a nonvanishing contribution. This condition yields local constraints on the occupation numbers $h_{\alpha \beta}^{a b}(t)$ and $m_{\alpha \beta}^{a b}(t)$ separately at each site $t$,

$$
\begin{aligned}
\sum_{\alpha, a}\left(h_{\alpha \beta}^{a b}(t-1)+m_{\alpha \beta}^{a b}(t)\right) & =1 \quad \forall \beta, b, t, \\
\sum_{\beta, b}\left(h_{\alpha \beta}^{a b}(t)+m_{\alpha \beta}^{a b}(t)\right) & =1 \quad \forall \alpha, a, t .
\end{aligned}
$$

The integration over the fermion fields is then replaced by a summation over all configurations of occupation numbers satisfying the constraints above.

The various configurations of occupation numbers and the corresponding constraints can most easily be specified graphically by representing each pair $\bar{\psi}_{\alpha}^{a}(t) \psi_{\alpha}^{a}(t)$ by a point $\bullet$ and each occupation number $h_{\alpha \beta}^{a b}(t), m_{\alpha \beta}^{a b}(t)$ by an arrow $\longrightarrow$ pointing from point $(a, \alpha)$ to $(b, \beta)$ saturating $\bar{\psi}_{\alpha}^{a}$ and $\psi_{\beta}^{b}$, respectively. The graphical building blocks are then simply given by the spatial (flavour or colour) hops characterised by $m_{\alpha \beta}^{a b}(t)=1$, cf. figure 1 , and the temporal hops characterised by $h_{\alpha \beta}^{a b}(t)=1$, cf. figure 2 , where the gauge links are reponsible for changing the flavour or colour index from $a$ to $b$. Due to the breaking of the time inversion symmetry, or equivalently charge conjugation, by the Wilson term 


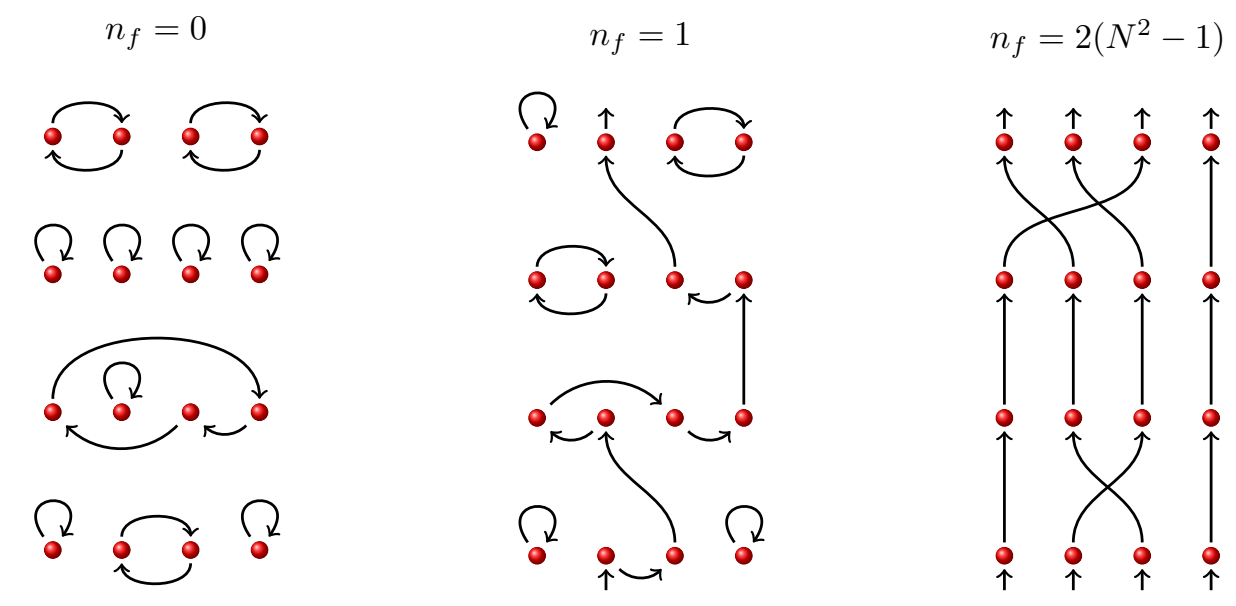

Figure 3. Three sample configurations of closed oriented loops for four fermionic degrees of freedom (representative for the generic $2\left(N^{2}-1\right)$ ones) on a periodic lattice with four time slices.

there exist only temporal hops in forward direction of time. The contribution of each local fermion integration can be read off from eq. (4.2) and are given as the weights in figures 1 and 2. From the contraints in eq. (4.3) and (4.4) it becomes immediately clear that in the graphical representation only closed, oriented fermion loops are allowed. Moreover, each fermion loop picks up the usual factor $(-1)$ from the Grassmann integration. Eventually, the full partition function in the fermion loop formulation reads

$$
Z=\int \mathcal{D} U \mathcal{D} X e^{-S_{B}\left[U, X_{i}\right]} \sum_{\{h, m\}} \prod_{t}\left[\left(W_{\alpha \beta}^{a b}\right)^{h_{\alpha \beta}^{a b}(t)}\left(\Phi_{\alpha \beta}^{a b}(t)\right)^{m_{\alpha \beta}^{a b}(t)}\right]
$$

where the sum is over all combinations of occupation numbers satisfying eq. (4.3) and (4.4).

\section{$5 \quad$ Fermion sectors and transfer matrices}

In figure 3 we show three sample configurations consisting of closed oriented fermion loops for four fermionic degrees of freedom (representative for the generic $2\left(N^{2}-1\right)$ ones). One immediately notices that the configurations can be classified according to the number of fermions $n_{f}$ propagating forward in time. For the three examples depicted in figure 3 the fermion numbers are $n_{f}=0,1$ and 4 (i.e. $n_{f}=2\left(N^{2}-1\right)$ for the generic case), respectively. In each sector, the propagation of the $n_{f}$ fermions can be described by transfer matrices

$$
T_{n_{f}}(t)=T_{n_{f}}^{\Phi}\left(X_{i}(t)\right) \cdot T_{n_{f}}^{W}(U)
$$

where the first transfer matrix describes the various ways how to connect $n_{f}$ fermions entering at time $t$ with $n_{f}$ fermions exiting at $t$. It depends on the boson field configuration $X_{i}(t)$ through the Yukawa interactions matrix $\Phi(t)$ and hence depends on $t$. The second transfer matrix describes how to connect $n_{f}$ fermions exiting at $t$ and entering at $t+1$, and hence depends on the gauge field $U$ through $W$ in eq. (2.6). In uniform gauge, this transfer matrix has no time dependence. Then, for a given gauge and boson field background 
$\left\{U, X_{i}(t)\right\}$ the fermion contribution to the partition function in the sector with $n_{f}$ fermions is simply given by

$$
\operatorname{det} \mathcal{D}_{n_{f}}\left[U, X_{i}\right]=\operatorname{Tr}\left[\prod_{t=0}^{L_{t}-1} T_{n_{f}}(t)\right] .
$$

The full contribution is then obtained by adding up all these terms taking into account a factor $(\mp 1) e^{\mu L_{t}}$ for each fermion loop winding around the lattice in temporal direction, with the sign depending on whether periodic or antiperiodic boundary conditions are employed. The expression eventually reads

$$
\operatorname{det} \mathcal{D}_{p, a}\left[U, X_{i} ; \mu\right]=\sum_{n_{f}=0}^{2\left(N^{2}-1\right)}\left(\mp e^{\mu L_{t}}\right)^{n_{f}} \operatorname{Tr}\left[\prod_{t=0}^{L_{t}-1} T_{n_{f}}(t)\right]
$$

and can directly be compared with eq. (3.4).

Let us now look in more detail at the transfer matrices separately in each sector. First we note that the size of $T_{n_{f}}$ is given by the number of states in sector $n_{f}$, i.e.,

$$
n \equiv\left(\begin{array}{c}
2\left(N^{2}-1\right) \\
n_{f}
\end{array}\right) \text {. }
$$

The sectors with $n_{f}=0$ and $n_{f}=2\left(N^{2}-1\right)$ are therefore particularly simple since in these cases the transfer matrix is just $1 \times 1$. We will hence first discuss these two sectors, followed by the still rather simple sectors with $n_{f}=1$ and $n_{f}=2\left(N^{2}-1\right)-1$, before presenting the generic case for arbitrary values of $n_{f}$.

\subsection{Sector $n_{f}=0$}

For $n_{f}=0$ we see by inspection of the corresponding configuration in figure 3 that there is no gauge link dependence, and hence $T_{0}^{W}=1$, while the transfer matrix $T_{0}^{\Phi}(t)$ must contain the sum of the weights of all fermion loop configurations on a given time slice $t$. By doing so, we need to take care that each nontrivial fermion loop picks up the usual factor $(-1)$ from the Grassmann integration. It is not difficult to see that a given time slice configuration can be specified by a permutation $\sigma$ of the indices $i=1, \ldots, 2\left(N^{2}-1\right)$ labelling the fermionic degrees of freedom. Each cycle $(i j k \ldots l)$ in the permutation then corresponds to a sequence of indices characterising a specific fermion loop and its weight is given by $\Phi_{i j} \Phi_{j k} \ldots \Phi_{l i}$. The total sign of the configuration is given by including a factor $(-1)$ for each nontrivial cycle, i.e., counting whether the number of nontrivial cycles in the permutation is even or odd which corresponds to the parity of the permutation. Finally, the sum over all configurations amounts to summing up all permutations including the corresponding weights and the signs given by the parity of the permutation. This prescription is of course nothing else than the definition for the determinant, so the transfer matrix in the $n_{f}=0$ sector is simply given by

$$
T_{0}^{\Phi}(t)=\operatorname{det} \Phi(t)
$$

and the total fermion contribution factorises completely,

$$
\operatorname{det} \mathcal{D}_{n_{f}=0}\left[U, X_{i}\right]=\prod_{t=0}^{L_{t}-1} \operatorname{det} \Phi(t) .
$$


Comparing this with eq. (3.7) we obviously find complete agreement. In the fermion loop approach however it is evident from the beginning that the gauge link $U$ does not contribute in the $n_{f}=0$ sector.

\subsection{Sector $n_{f}=n_{f}^{\max }$}

For $n_{f}=2\left(N^{2}-1\right) \equiv n_{f}^{\max }$ the transfer matrix $T_{n_{f}^{\max }(t)}$ is again $1 \times 1$. While there are no contributions from the Yukawa interaction, hence $T_{n_{f}^{\max }}^{\Phi}(t)=1$, we need to take into account the nontrivial hopping in colour space. The complication arising here stems from the fact that depending on the number of hoppings in colour space, the total number of fermion loops winding in temporal direction changes, but not the number of winding fermions. For example, if there are only colour diagonal hops, the number of winding loops is $n_{f}^{\max }$ and the corresponding contribution comes with a positive sign. On the other hand, if there is one single nondiagonal colour hop two loops merge into one, so the number of winding loops becomes $n_{f}^{\max }-1$ and the contribution should hence contain a negative sign relative to the contribution with $n_{f}^{\max }$ loops. So for every nondiagonal colour hop the number of loops is changing by one.

Similarly to the $n_{f}=0$ sector we need to take all permutations of the colour indices $a, b$ into account. For each nontrivial permutation of two indices the number of fermion loops winding in temporal direction is reduced by one and we take this into account by including a factor $(-1)$. Summing over all permutations including the sign corresponding to the parity of the permutations again yields the determinant, i.e.,

$$
T_{n_{f}^{\max }}^{W}=\operatorname{det}[W]=1
$$

yielding the total contribution

$$
\operatorname{det} \mathcal{D}_{n_{f}^{\max }}\left[U, X_{i}\right]=\prod_{t=0}^{L_{t}-1} T_{n_{f}^{\max }}(t)=1 .
$$

This is in accordance with the result from the determinant reduction, cf. eq. (3.6), and it is obvious that the same result would be obtained without referring to a particular gauge. Since the fermions are completely saturated by the temporal hopping terms and contribute only trivially to the canonical determinant, this sector corresponds to the quenched one as noted before.

\subsection{Sector $n_{f}=1$}

Next, we look at the sector with $n_{f}=1$ fermions. The corresponding transfer matrices $T_{1}(t)$ are of size $2\left(N^{2}-1\right) \times 2\left(N^{2}-1\right)$. Each matrix element $\left(T_{1}^{\Phi}(t)\right)_{i j}$ contains the sum of weights of all configurations at fixed $t$ where the fermion degree of freedom $i=(a, \alpha)$ is entering time slice $t$ and $j=(b, \beta)$ is leaving. The corresponding degrees of freedom are then already saturated by the corresponding hops in and out of the time slice and hence the weights $\Phi_{k i}$ and $\Phi_{j k}, k=1, \ldots, 2\left(N^{2}-1\right)$ can not appear in any of the configurations. The remaining time slice configurations can be obtained in analogy to the considerations in the $n_{f}=0$ sector, that is by constructing all permutations, i.e., cycles of the remaining degrees 
of freedom and taking into account factors of $(-1)$ for each nontrivial cycle. Following the arguments from the $n_{f}=0$ sector it turns out that this is again equivalent to taking the determinant of $\Phi(t)$, but with row $j$ and column $i$ removed, i.e.,

$$
\left(T_{1}^{\Phi}\right)_{i j}=\left.(-1)^{i+j} \operatorname{det} \Phi\right|_{\Phi_{k i}=\delta_{k j}, \Phi_{j k}=\delta_{i k}} \equiv(-1)^{i+j} \operatorname{det} \Phi \text { iो }^{\text {i }}
$$

which is in fact the $(j, i)$-cofactor of $\Phi$. This will be discussed in more detail in section 5.5. Similarly, in order to include the colour changing hops due to the gauge link between time slices we multiply with the corresponding gauge link transfer matrix $T_{1}^{W}$

$$
\left(T_{1}^{W}\right)_{i j}=(W)_{i j}
$$

which in uniform gauge is constant in time and is in fact the complementary $(i, j)$-minor $\operatorname{det} W^{i j}$. Eventually, the full fermion contribution in the $n_{f}=1$ sector reads

$$
\operatorname{det} \mathcal{D}_{n_{f}=1}\left[U, X_{i}\right]=\operatorname{Tr} \prod_{t=0}^{L_{t}-1}\left[T_{1}^{\Phi}(t) \cdot T_{1}^{W}\right]
$$

and comparing this result to the one in eq. (3.8) from the fugacity expansion, we find a nontrivial relationship between the two expressions. We will comment further on this relation in section 5.5 and establish it in detail in appendix B.

\subsection{Sector $n_{f}=n_{f}^{\max }-1$}

In the sector where all but one, i.e., $n_{f}^{\max }-1$ fermions are propagating, the states of the transfer matrices $T_{n_{f}^{\max }-1}(t)$ are most conveniently labelled by the degree of freedom $i=(a, \alpha)$ not occupied by a temporal hopping term. The transfer matrices are hence of size $2\left(N^{2}-1\right) \times 2\left(N^{2}-1\right)=n_{f}^{\max } \times n_{f}^{\max }$. The matrix elements $\left(T_{n_{f}^{\max }-1}^{\Phi_{i j}}\right)_{i j}$ are calculated following the arguments outlined above for the $n_{f}=0$ and 1 sector, namely to take the determinant of the Yukawa matrix $\Phi$ with all columns and rows deleted except $i$ and $j$, respectively. The reduced Yukawa matrix is then just a single element and hence we have

$$
\left(T_{n_{f}^{\max }-1}^{\Phi}\right)_{i j}=(-1)^{i+j} \Phi_{i j}
$$

which is just the complementary $(i, j)$-cofactor of $\Phi$ up to an overall sign. The transfer matrix describing all the possible configurations within a time slice needs to be complemented by the one inducing the colour changing hops due to the gauge link between the time slices. If fermion $i$ is not hopping out of $t$ and $j$ not into $t+1$ they will not contribute, while the mixing of the remaining degrees of freedom is described as before by taking the determinant of the hop matrix,

$$
\left(T_{n_{f}^{\max }-1}^{W}\right)_{i j}=\operatorname{det} W^{\text {iो }}
$$

which is the $(i, j)$-minor of $W$. The full fermion contribution in the $n_{f}=n_{f}^{\max }-1$ sector finally yields

$$
\operatorname{det} \mathcal{D}_{n_{f}^{\max }-1}\left[U, X_{i}\right]=\operatorname{Tr} \prod_{t=0}^{L_{t}-1}\left[T_{n_{f}^{\max }-1}^{\Phi}(t) \cdot T_{n_{f}^{\max }-1}^{W}\right] .
$$

This can be compared to the one in eq. (3.9) from the fugacity expansion and we find again a nontrivial relationship between the two expressions. 


\subsection{Sector with generic $n_{f}$}

Similar constructions can be worked out in all the other sectors, but the constructions become more involved since the number of states grows rapidly towards the half-filled sector with $n_{f}=2\left(N^{2}-1\right) / 2$. However, our previous discussion indicates a generic pattern which becomes clear after careful further investigation of all the weights and signs of each configuration. Employing some higher linear algebra one can eventually formulate the following rule. The sector with $n_{f}$ fermions contains $n=\left(\begin{array}{c}n_{f}^{\max } \\ n_{f}\end{array}\right)$ states and the elements of the corresponding $n \times n$ transfer matrix $T_{n_{f}}^{\Phi}$ are given by the cofactors of $\Phi$ of order $n_{f}$, while the matrix elements of $T_{n_{f}}^{W}$ are given by the complementary minors of $W$.

To be more precise, let $A$ and $B$ be two index sets $A, B \subseteq\left\{1,2, \ldots, 2\left(N^{2}-1\right)\right\}$ of size $n_{f}$, then the cofactor of $\Phi$ of order $n_{f}$ is the signed determinant of the $\left(2\left(N^{2}-1\right)-n_{f}\right) \times$ $\left(2\left(N^{2}-1\right)-n_{f}\right)$ submatrix $\Phi^{\mathrm{B} X}$ obtained from $\Phi$ by deleting the rows indexed by $B$ and the columns indexed by $A$, so

$$
\left(T_{n_{f}}^{\Phi}\right)_{A B}=(-1)^{p(A, B)} \operatorname{det} \Phi^{\mathcal{X} \backslash}
$$

where $p(A, B)=\sum_{i \in A} i+\sum_{j \in B} j$, while the complementary minor $\operatorname{det} W^{A B}$ is the determinant of the $n_{f} \times n_{f}$ submatrix $W^{A B}$ obtained from $W$ by keeping only the rows indexed by $A$ and the columns indexed by $B$,

$$
\left(T_{n_{f}}^{W}\right)_{A B}=\operatorname{det} W^{A B}
$$

If the two sets $A$ and $B$ are equal, the cofactors reduce to minors and the corresponding determinants are called principal minors or principal complementary minors. Note also that in the literature the role of the minor and complementary minor is sometimes exchanged.

In analogy to the discussion before, the cofactor $C_{\mathcal{B} \backslash}(\Phi)=(-1)^{p(A, B)} \operatorname{det} \Phi^{\mathcal{B} \backslash}$ includes all contributions to the transition of $n_{f}$ fermions indexed by $A$ entering at time $t$ to $n_{f}$ fermions indexed by $B$ exiting from time $t$, with all the weights and signs properly accounted for. Similarly, the minor $M_{A B}(W)=\operatorname{det} W^{A B}$ connects $n_{f}$ fermions indexed by $A$ exiting $t$ in all possible ways with $n_{f}$ fermions indexed by $B$ entering time $t+1$ with the correct weight and sign for each connection. Hence, the full transfer matrix at time $t$ in the sector with $n_{f}$ fermions is then $T_{n_{f}}^{\Phi}(t) \cdot T_{n_{f}}^{W}$ and the corresponding canonical determinant reads

$$
\operatorname{det} \mathcal{D}_{n_{f}}\left[U, X_{i}\right]=\operatorname{Tr} \prod_{t=0}^{L_{t}-1}\left[T_{n_{f}}^{\Phi}(t) \cdot T_{n_{f}}^{W}\right] .
$$

It is easy to check that this generic definition yields the correct expressions for the transfer matrices and canonical determinants for the cases $n_{f}=0,1, n_{f}^{\max }-1, n_{f}^{\max }$ discussed in the previous sections. Note that for the empty sets $A=B=\{\}$ the principal minor, and analogously the complementary principal minor for the full sets $A=B=\left\{1, \ldots, 2\left(N^{2}-1\right)\right\}$, is 1 by definition.

Finally, one can show that the canonical determinants obtained in the fermion loop approach are equal to the ones using the fermion matrix reduction, cf. eq. (3.11). Using 
various relations between matrices of minors and cofactors, one can derive that

$$
\left(\prod_{t=0}^{L_{t}-1}\left[T_{n_{f}}^{\Phi}(t) \cdot T_{n_{f}}^{W}\right]\right)_{A B}=(-1)^{p(A, B)} \operatorname{det} \mathcal{T}^{\not \backslash \mathbb{B}}=C_{\mathbf{X} \mathcal{B}}(\mathcal{T}) .
$$

The details of this derivation are given in appendix B. The r.h.s. forms the $n \times n$ matrix of cofactors of $\mathcal{T}$ of order $n_{f}$ and the trace in eq. (5.17) then yields the sum over the $n$ principal minors of $\mathcal{T}$ of order $n_{f}$ denoted by $E_{n_{f}}$, i.e.,

$$
\operatorname{det} \mathcal{D}_{n_{f}}=\sum_{B} \operatorname{det} \mathcal{T}^{\mathcal{B} \backslash} \equiv E_{n_{f}}(\mathcal{T})
$$

Recalling a known relation from linear algebra between the sum of minors of a matrix and its symmetric functions [31] one has

$$
E_{n_{f}}(\mathcal{T})=S_{n_{f}^{\max }-n_{f}}(\mathcal{T})
$$

which establishes the equivalence between eq. (3.11) and eq. (5.19).

\subsection{Remarks}

We close this section with several remarks. Firstly, we note that in contrast to the full determinant $\operatorname{det}\left[U, X_{i}\right]$, which can be proven to be positive [15], the various canonical determinants $\operatorname{det} \mathcal{D}_{n_{f}}\left[U, X_{i}\right]$ need not necessarily be positive. Obviously, $\operatorname{det} \mathcal{D}_{n_{f}=2\left(N^{2}-1\right)}\left[U, X_{i}\right]$ is so and it seems that at least $\operatorname{det} \mathcal{D}_{n_{f}=0}\left[U, X_{i}\right]$ is also positive, although we do not have any proof. It would be interesting to study potential fermion sign problems in the canonical sectors in the present model. Despite its simplicity due to the low dimension, it nevertheless contains all the important features of a gauge theory, and hence conclusions can most likely be generalised to more complicated gauge theories in higher dimensions, such as QCD in the canonical formulation [29].

Secondly, we note that the various sectors, in particular the ones with many fermions, can in principle be simulated by open fermion string (fermion worm) algorithms along the lines described in $[23,24]$. This approach has indeed already been applied successfully in ordinary supersymmetric quantum mechanics [22], in the supersymmetric nonlinear $\mathrm{O}(N)$ sigma model [32] and in the two dimensional $\mathcal{N}=1$ Wess-Zumino model [25, 27] where the transfer matrix techniques discussed here and in [26] are out of reach. Furthermore, for the model discussed in this paper, a discrete bond formulation for the bosonic degrees of freedom is available [33]. Such a formulation promises a huge gain in efficiency for numerical simulations, but it is not clear whether the bosonic bond formulation can be put into practice.

Thirdly, from investigations in the Hamiltonian formulation of the theory [3], where time is treated as a continuous variable, it is known that there is a (spectral) symmetry between the sectors with $n_{f}$ and $2\left(N^{2}-1\right)-n_{f}$ fermions, due to the exchange symmetry between particles and antiparticles. Our results above indicate that the symmetry is not maintained by our choice of the discretisation in the Lagrangian formalism, but the reason for this is clear. As we mentioned earlier the Wilson term needed to control the doubler 
fermions explicitly breaks the time reversal and hence the charge conjugation symmetry which of course is crucial for an exact particle/antiparticle exchange symmetry. However, since the symmetries are restored in the continuum limit without fine tuning, the symmetries between the various canonical sectors will also be mended automatically in the continuum, and the difference between the related sectors will provide a good estimate of the remaining systematic lattice artefacts.

\section{Conclusions and outlook}

In this paper we have investigated in detail the structure of the fermionic part of the $d=4$ dimensional supersymmetric Yang-Mills quantum mechanics, i.e., $\mathcal{N}=4$ SYM QM with gauge group $\mathrm{SU}(N)$. On the one hand, we derived a reduced fermion matrix whose size is independent of the temporal extent of the lattice. In addition, the dependence on the chemical potential is factored out and this allows the exact projection of the fermion determinant onto the canonical sectors with fixed fermion number, once the eigenvalues of the reduced matrix are calculated. On the other hand, we have presented the fermion loop formulation of the theory in which the grand canonical fermion determinant naturally decomposes into sectors with fixed fermion numbers. The construction of transfer matrices is rather straightforward in the various fermion sectors and the comparison with the fugacity expansion, accessible via the reduced fermion matrix, yields identical results and interesting relations between the transfer matrices and the eigenvalues of the reduced fermion matrix. In fact, we presented a proof which establishes the equivalence of the canonical determinants from the reduced fermion matrix approach and from the fermion loop formulation on the algebraic level.

Our results open various possibilities for a range of nonperturbative investigations of the theory. This can be done for example by numerical simulations using methods different from the usual Hybrid Monte Carlo approach, either using the transfer matrices in the various canonical sectors with fixed fermion numbers, or using the projection to the sectors with the help of the reduced fermion matrix. Another interesting approach could be the application of mean field methods to the spatial gauge degrees of freedom, again either in the transfer matrix approach or using the reduced fermion matrix. It is even conceivable that the methods presented here and the emerged simple structures lead to new analytic results in some interesting limits. All results obtained either way will provide important insights into the conjectured M-theory and will add to our understanding of the corresponding gauge/gravity duality, besides unveiling interesting physics of the model itself.

Another interesting line of research starting from here concerns the investigation of ordinary, non-supersymmetric gauge field theories in higher dimensions at finite fermion density, such as QCD at finite baryon density. It is notoriously difficult to obtain reliable results in these theories using the known numerical approaches, due to the intrinsic fermion sign problem at finite density, and any insight into how the simulations of these theories could be facilitated would be extremely valuable. The explicit fugacity expansion derived in this paper allows to investigate finite density simulations or canonical simulations in a 
simple setup which nevertheless displays a similar structure, and hence contains all the important features, as the more complicated theories in higher dimensions such as QCD.

Finally, the extension of the loop formulation to $\mathcal{N}=16$ supersymmetric Yang-Mills quantum mechanics is in principle straightforward but requires special care. This is due to the fact that the corresponding dimensionally reduced model has obviously a different Dirac structure, and it remains to be seen whether the structure is compatible with the requirements for the fermion loop formulation. The fermion matrix reduction on the other hand should be unaffected by the change of the Dirac structure.

\section{Acknowledgments}

We thank Dan Boss, Adolpho Guarino, Piotr Korcyl, Andreas Wipf and Jacek Wosiek for useful discussions, and in particular Piotr Korcyl and Dan Boss for sharing their codes for the $d=4$ dimensional $\mathrm{SU}(N)$ SYM QM with $N=2$ and $N=2,3$, respectively.

\section{A Determinations of canonical determinants}

In this appendix we review three alternative methods to calculate the canonical determinants from the matrix $\mathcal{T}$ in eq. (3.3). As shown in section 3 the canonical determinants are just the coefficients of the characteristic polynomial of the matrix $\mathcal{T}$. The first method provides recursion relations which yield the coefficients in terms of the eigenvalues $\tau_{i}$ of $\mathcal{T}$. The second method evaluates the coefficients in terms of the traces of powers of $\mathcal{T}$ and the third makes use of the minors of $\mathcal{T}$. The latter method turns out to be closely related to the transfer matrix approach in the fermion loop formulation and hence deserves special emphasis.

In the following we assume the matrix $\mathcal{T}$ to be of size $n \times n$ and for simplicity we consider only the case of antiperiodic b.c., hence the relevant characteristic polynomial is

$$
g(x)=\operatorname{det}(\mathcal{T}+x \cdot \mathbb{1})=\sum_{k=0}^{n} c_{k} \cdot x^{k}
$$

where $\mathbb{1}$ is the $n \times n$ unit matrix and the coefficients $c_{k}$ are the canonical determinants $\operatorname{det} \mathcal{D}_{n_{f}=k}$ in sector $k$.

\section{A.1 Coefficients from recursion relations}

The coefficients can be obtained from the eigenvalues $\tau_{i}$ of $\mathcal{T}$ using recursive relations [29]. To this end, we first define the partial products

$$
\Pi_{r}(x)=\prod_{j=1}^{r}\left(\tau_{j}+x\right)=\sum_{k=1}^{r} c_{k}^{(r)} x^{k}
$$

which fulfill $\prod_{r+1}(x)=\left(\tau_{r+1}+x\right) \prod_{r}(x)$. Setting $c_{-1}^{(r)}=0$ we have the recursion relation

$$
c_{k}^{(r+1)}=\tau_{r+1} c_{k}^{(r)}+c_{k-1}^{(r)}
$$


for all $0 \leq k \leq r+1$ which allows to compute $c_{k}^{(r+1)}$ from $c_{k}^{(r)}$. After $n$ steps we obtain the coefficients $c_{k} \equiv c_{k}^{(n)}$ of $\prod_{n}(x)$ which are then just the canonical determinants $\operatorname{det} \mathcal{D}_{n_{f}=k}$. The generalisation of the recursion to include the minus sign from the periodic b.c. is straightforward.

\section{A.2 Coefficients in terms of traces}

Here we review the calculation of the coefficients $c_{k}$ in terms of traces of powers of the matrix $\mathcal{T}$. To do so we introduce the notation

$$
t_{k}=\operatorname{Tr}\left(\mathcal{T}^{k}\right) .
$$

Then, Newton's identities (or the Newton-Girard formulae) provide a set of relations between the traces,

$$
t_{1}-c_{n-1}=0, \quad t_{k}-c_{n-1} t_{k-1}+\ldots-c_{n-k+1} t_{1}+k \cdot c_{n-k}=0, \quad k=2,3, \ldots, n,
$$

which can be solved recursively. The solution can conveniently be written down in closed form as

$$
c_{n-k}=\frac{1}{k !} \operatorname{det}\left(\begin{array}{cccccc}
t_{1} & 1 & 0 & 0 & \cdots & 0 \\
t_{2} & t_{1} & 2 & 0 & \cdots & 0 \\
t_{3} & t_{2} & t_{1} & 3 & \cdots & 0 \\
\vdots & \vdots & \vdots & \vdots & \ddots & \vdots \\
t_{k-1} & t_{k-2} & t_{k-3} & t_{k-4} & \cdots & k-1 \\
t_{k} & t_{k-1} & t_{k-2} & t_{k-3} & \cdots & t_{1}
\end{array}\right)
$$

and the generalisation to periodic b.c. is again straighforward.

\section{A.3 Coefficients in terms of minors}

Instead of computing the traces of the matrices $\mathcal{T}, \mathcal{T}^{2}, \mathcal{T}^{3}, \ldots, \mathcal{T}^{n}$ we now present an alternative method for determining the coefficients of the characteristic polynomial which is more interesting from the point of view of the transfer matrix construction discussed in section 5. The method involves the expansion of determinants of order 1 to $n$ [34]. In order to determine the coefficients $c_{k}$ of $x^{k}$ in $g(x)$ it is useful to separate the occurrences of $x$ by introducing

$$
f\left(x_{1}, x_{2}, \ldots, x_{n}\right)=\operatorname{det}\left(\mathcal{T}+\operatorname{diag}\left(x_{1}, x_{2}, \ldots x_{n}\right)\right) .
$$

One then has $g(x)=f(x, x, \ldots, x)$ and $c_{k}$ is the sum of the coefficients of the terms with total degree $k$ in $f\left(x_{1}, x_{2}, \ldots, x_{n}\right)$. Since $f\left(x_{1}, x_{2}, \ldots, x_{n}\right)$ is of degree 1 in each $x_{i}$, it is straightforward to express the coefficient in terms of derivatives w.r.t. $x_{i}$ 's,

$$
c_{k}=\left.\sum_{1 \leq i_{1}<\cdots<i_{k} \leq n} \frac{\partial^{k}}{\partial x_{i_{1}} \partial x_{i_{2}} \cdots \partial x_{i_{k}}} f\left(x_{1}, x_{2}, \ldots, x_{n}\right)\right|_{x_{1}=x_{2}=\ldots=x_{n}=0}
$$

where $0 \leq k \leq n$. As a consequence the coefficients are now expressed explicitly in terms of the matrix elements of $\mathcal{T}$. Denoting them by $t_{i j}$ it turns out that

$$
c_{k}=\sum_{1 \leq i_{1}<\cdots<i_{k} \leq n} \frac{\partial^{k}}{\partial t_{i_{1} i_{1}} \partial t_{i_{2} i_{2}} \cdots \partial t_{i_{k} i_{k}}} \operatorname{det} \mathcal{T} .
$$


This can be seen most easily by suppressing the dependence of $\operatorname{det} \mathcal{T}$ on the off-diagonal elements $t_{i j}, i \neq j$ and define $D$ as a function of the $n$ variables $t_{11}, t_{22}, \ldots, t_{n n}$,

$$
D\left(t_{11}, t_{22}, \ldots, t_{n n}\right) \equiv \operatorname{det} \mathcal{T}
$$

and hence

$$
f\left(x_{1}, x_{2}, \ldots, x_{n}\right)=D\left(t_{11}+x_{1}, t_{22}+x_{2}, \ldots, t_{n n}+x_{n}\right) .
$$

It is then immediately clear that

$$
\frac{\partial^{k} f}{\partial x_{i_{1}} \partial x_{i_{2}} \ldots \partial x_{i_{k}}}=\frac{\partial^{k} D\left(t_{11}+x_{1}, t_{22}+x_{2}, \ldots, t_{n n}+x_{n}\right)}{\partial t_{i_{1} i_{1}} \partial t_{i_{2} i_{2}} \cdots \partial t_{i_{k} i_{k}}} .
$$

from which eq. (A.9) follows via eq. (A.10).

On the other hand the rules for the Laplace expansion of a determinant by a row or a column indicate that $\partial \operatorname{det} \mathcal{T} / \partial t_{i j}$ is the $(i, j)$-cofactor of $\mathcal{T}$, or in fact the $(i, i)$-minor when $i=j$. Therefore, the partial derivatives in eq. (A.9) are simply the subdeterminants of $\mathcal{T}$ resulting from crossing out the rows and columns numbered by $i_{1}, i_{2}, \ldots, i_{k}$, i.e., the principal minors of $\mathcal{T}$ of order $k$.

Denoting the sum of principal minors of order $k$ of $\mathcal{T}$ by $E_{k}(\mathcal{T})$ and keeping in mind that $\operatorname{det} \mathcal{D}_{k}=c_{k}$ one finds by comparison with eq. (3.11) that

$$
S_{n-k}(\mathcal{T})=E_{k}(\mathcal{T})
$$

for each $k=1, \ldots, n$, which is a known identity in matrix analysis from linear algebra, see e.g. [31].

Comparing these results with the ones derived in section 5 we immediately notice that the trace over the states of the transfer matrix is represented in eq. (A.9) by the sum $\sum_{i_{1}<i_{2}<\ldots<i_{k}}$. The number of summands here is $\left(\begin{array}{l}n \\ k\end{array}\right)$ and indeed equal to the number of states in the sector with $n_{f}=k$. Furthermore, the principal subdeterminants (minors) in eq. (A.9) correspond to the diagonal elements of the product of transfer matrices in the given sector.

\section{B Equivalence of canonical determinants}

Here we show that the canonical determinants obtained in the fermion loop approach, cf. eq. (5.17), are equal to the ones using the fermion matrix reduction, cf. eq. (3.11).

Following the notation introduced in section 5.5, for two index sets $A$ and $B$ of size $n_{f}$ the transfer matrix $T_{n_{f}}^{\Phi}$ in eq. (5.15) is the transposed matrix of cofactors of $\Phi$ of order $n_{f}$ and is denoted by

$$
\left(T_{n_{f}}^{\Phi}\right)_{A B}=C_{B \backslash X}(\Phi)
$$

while the transfer matrix $T_{n_{f}}^{W}$ in eq. (5.16) is the matrix of complementary minors denoted by

$$
\left(T_{n_{f}}^{W}\right)_{A B}=M_{A B}(W)
$$


Now we note that the complementary minor matrix $M_{A B}(W)$ is related to the minor matrix of the inverse $M_{\mathbb{A}_{\mathcal{B}}}\left(W^{-1}\right)$ by

$$
M_{\mathrm{X} 及}\left(W^{-1}\right)=(-1)^{p(A, B)} \frac{M_{B A}(W)}{\operatorname{det} W}
$$

where $p(A, B)=\sum_{i \in A} i+\sum_{j \in B} j$. Up to the determinant, the r.h.s. is the higher order generalisation of the adjugate (or classical adjoint) of $W$, i.e. $\operatorname{Adj}_{A B}(W)$. (To order 1 the adjugate is just the transposed complementary cofactor matrix.) Hence, with $\operatorname{det} W=1$, $W^{-1}=W^{\dagger}=W^{T}$ and $M_{A B}(W)=M_{B A}\left(W^{T}\right)$ we have

$$
C_{\mathrm{B} \backslash \mathrm{X}}\left(W^{\dagger}\right)=C_{\chi_{\mathcal{B}}(W)}\left(W M_{A B}(W),\right.
$$

i.e., the transfer matrix $T_{n_{f}}^{W}$ can be expressed as a cofactor matrix instead of a complementary minor matrix.

Next, we note that the cofactor matrix $C$ and the corresponding minor matrix $M$ are related by modifying the sign of each element according to $C_{A B}=(-1)^{p(A, B)} M_{A B}$. The sign change can be achieved by a similarity transformation with the matrix $S_{A B}=$ $(-1)^{\sum_{i \in A} i} \delta_{A B}$, i.e., $C=S^{-1} \cdot M \cdot S$. Therefore a product of cofactor matrices becomes a product of minor matrices under a trace, and so we can eventually write

$$
\begin{aligned}
\operatorname{det} \mathcal{D}_{n_{f}} & =\operatorname{Tr} \prod_{t=0}^{L_{t}-1}\left[T_{n_{f}}^{\Phi}(t) \cdot T_{n_{f}}^{W}\right] \\
& =\operatorname{Tr} \prod_{t=0}^{L_{t}-1}\left[C(\Phi(t))^{T} \cdot C(W)\right] \\
& =\operatorname{Tr} \prod_{t=0}^{L_{t}-1}[M(\Phi(t)) \cdot M(W)] .
\end{aligned}
$$

Note that we have made use of the fact that $C(\Phi)^{\dagger}=C(\Phi)$ since $\Phi^{\dagger}=\Phi$.

We can now employ the Cauchy-Binet formula which states in its symmetric form that given the $n \times n$ matrices $P, Q$ with $R=P Q$ and two index sets $A, B$ of size $1 \leq k \leq n$ the $(A B)$-minor of $R$ is

$$
\operatorname{det} R^{X B}=\sum_{D} \operatorname{det} P^{X \bar{X}} \operatorname{det} Q^{\mathrm{D} B}
$$

where the sum is taken over all index sets $D$ of size $k$. From the formula it follows that for the matrices of minors (and similarly for the matrices of cofactors) one has

$$
M(P Q)=M(P) M(Q)
$$

and consequently from eq. (B.7)

$$
\begin{aligned}
\operatorname{det} \mathcal{D}_{n_{f}} & =\operatorname{Tr} \prod_{t=0}^{L_{t}-1}[M(\Phi(t)) \cdot M(W)] \\
& =\operatorname{Tr} M\left(\prod_{t=0}^{L_{t}-1}[\Phi(t) W]\right) \\
& =\operatorname{Tr} M(\mathcal{T}) .
\end{aligned}
$$


Finally, the trace sums over the $\left(\begin{array}{c}n_{f}^{\max } \\ n_{f}\end{array}\right)$ diagonal elements of the minor matrix which are just the principal minors,

$$
\operatorname{det} \mathcal{D}_{n_{f}}=\sum_{B} \operatorname{det} \mathcal{T}^{\mathcal{B} \mathcal{B}} \equiv E_{n_{f}}(\mathcal{T})
$$

Recalling from linear algebra [31] the fact that the sum of all principal minors of order $n_{f}$ of a matrix is equal to the $\left(n_{f}^{\max }-n_{f}\right)^{t h}$ symmetric function of its eigenvalues, i.e. $E_{n_{f}}(\mathcal{T})=$ $S_{n_{f}^{\max }-n_{f}}(\mathcal{T})$, eventually proves the equivalence between $\operatorname{det} \mathcal{D}_{n_{f}}$ from the fermion loop formulation in eq. (5.19) and from the fermion matrix reduction in eq. (3.11).

Open Access. This article is distributed under the terms of the Creative Commons Attribution License (CC-BY 4.0), which permits any use, distribution and reproduction in any medium, provided the original author(s) and source are credited.

\section{References}

[1] T. Banks, W. Fischler, S.H. Shenker and L. Susskind, M-theory as a matrix model: $a$ conjecture, Phys. Rev. D 55 (1997) 5112 [hep-th/9610043] [INSPIRE].

[2] W. Taylor, M(atrix) theory: matrix quantum mechanics as a fundamental theory, Rev. Mod. Phys. 73 (2001) 419 [hep-th/0101126] [INSPIRE].

[3] J. Wosiek, Spectra of supersymmetric Yang-Mills quantum mechanics, Nucl. Phys. B 644 (2002) 85 [hep-th/0203116] [INSPIRE].

[4] M. Campostrini and J. Wosiek, High precision study of the structure of $D=4$ supersymmetric Yang-Mills quantum mechanics, Nucl. Phys. B 703 (2004) 454 [hep-th/0407021] [INSPIRE].

[5] G. Veneziano and J. Wosiek, Planar quantum mechanics: an intriguing supersymmetric example, JHEP 01 (2006) 156 [hep-th/0512301] [INSPIRE].

[6] G. Veneziano and J. Wosiek, A supersymmetric matrix model. II. Exploring higher-fermion-number sectors, JHEP 10 (2006) 033 [hep-th/0607198] [INSPIRE].

[7] G. Veneziano and J. Wosiek, A supersymmetric matrix model. III. Hidden SUSY in statistical systems, JHEP 11 (2006) 030 [hep-th/0609210] [INSPIRE].

[8] M. Campostrini and J. Wosiek, Exact Witten index in D=2 supersymmetric Yang-Mills quantum mechanics, Phys. Lett. B 550 (2002) 121 [hep-th/0209140] [INSPIRE].

[9] P. Korcyl, Solutions of $D=2$ supersymmetric Yang-Mills quantum mechanics with $\mathrm{SU}(N)$ gauge group, J. Math. Phys. 52 (2011) 052105 [arXiv:1101.0591] [INSPIRE].

[10] P. Korcyl, Analytic calculation of Witten index in D $=2$ supersymmetric Yang-Mills quantum mechanics, J. Math. Phys. 53 (2012) 102102 [arXiv:1101.0668] [InSPIRE].

[11] P. Korcyl, Gauge invariant plane-wave solutions in supersymmetric Yang-Mills quantum mechanics, J. Math. Phys. 52 (2011) 042102 [arXiv: 1008.2975] [INSPIRE].

[12] P. Korcyl, Exact solutions to D = 2 supersymmetric Yang-Mills quantum mechanics with SU(3) gauge group, Acta Phys. Polon. Supp. 2 (2009) 623 [arXiv:0911.2152] [InSPIRE].

[13] R.A. Janik and J. Wosiek, Towards the matrix model of M-theory on a lattice, Acta Phys. Polon. B 32 (2001) 2143 [hep-th/0003121] [InSPIRE].

[14] N. Kawahara, J. Nishimura and S. Takeuchi, Phase structure of matrix quantum mechanics at finite temperature, JHEP 10 (2007) 097 [arXiv:0706.3517] [INSPIRE]. 
[15] S. Catterall and T. Wiseman, Towards lattice simulation of the gauge theory duals to black holes and hot strings, JHEP 12 (2007) 104 [arXiv:0706.3518] [INSPIRE].

[16] S. Catterall and T. Wiseman, Black hole thermodynamics from simulations of lattice Yang-Mills theory, Phys. Rev. D 78 (2008) 041502 [arXiv: 0803.4273] [InSPIRE].

[17] S. Catterall and T. Wiseman, Extracting black hole physics from the lattice, JHEP 04 (2010) 077 [arXiv: 0909.4947] [INSPIRE].

[18] M. Hanada, J. Nishimura and S. Takeuchi, Non-lattice simulation for supersymmetric gauge theories in one dimension, Phys. Rev. Lett. 99 (2007) 161602 [arXiv:0706.1647] [InSPIRE].

[19] K.N. Anagnostopoulos, M. Hanada, J. Nishimura and S. Takeuchi, Monte Carlo studies of supersymmetric matrix quantum mechanics with sixteen supercharges at finite temperature, Phys. Rev. Lett. 100 (2008) 021601 [arXiv:0707.4454] [INSPIRE].

[20] M. Hanada, J. Nishimura, Y. Sekino and T. Yoneya, Direct test of the gauge-gravity correspondence for matrix theory correlation functions, JHEP 12 (2011) 020 [arXiv: 1108.5153] [INSPIRE].

[21] M. Honda, G. Ishiki, S.-W. Kim, J. Nishimura and A. Tsuchiya, Direct test of the AdS/CFT correspondence by Monte Carlo studies of $N=4$ super Yang-Mills theory, JHEP 11 (2013) 200 [arXiv: 1308.3525] [INSPIRE].

[22] D. Baumgartner and U. Wenger, Simulation of supersymmetric models on the lattice without a sign problem, PoS (Lattice 2010) 245 [arXiv: 1104.0213] [INSPIRE].

[23] U. Wenger, Efficient simulation of relativistic fermions via vertex models, Phys. Rev. D 80 (2009) 071503 [arXiv:0812.3565] [INSPIRE].

[24] U. Wenger, Simulating Wilson fermions without critical slowing down, PoS(LAT2009) 022 [arXiv: 0911.4099] [INSPIRE].

[25] D. Baumgartner, K. Steinhauer and U. Wenger, Supersymmetry breaking on the lattice: the $N=1$ Wess-Zumino model, PoS (LATTICE 2011) 253 [arXiv:1111.6042] [INSPIRE].

[26] D. Baumgartner and U. Wenger, Exact results for supersymmetric quantum mechanics on the lattice, PoS (LATTICE 2011) 239 [arXiv:1201.1485] [InSPIRE].

[27] D. Baumgartner, K. Steinhauer and U. Wenger, Spontaneous supersymmetry breaking in the $2 d N=1$ Wess-Zumino model, PoS (LATTICE 2012) 043 [arXiv:1311.5089] [INSPIRE].

[28] P. Hasenfratz and F. Karsch, Chemical potential on the lattice, Phys. Lett. B 125 (1983) 308 [INSPIRE].

[29] A. Alexandru and U. Wenger, QCD at non-zero density and canonical partition functions with Wilson fermions, Phys. Rev. D 83 (2011) 034502 [arXiv: 1009.2197] [INSPIRE].

[30] G. Bergner, T. Kaestner, S. Uhlmann and A. Wipf, Low-dimensional supersymmetric lattice models, Annals Phys. 323 (2008) 946 [arXiv:0705.2212] [INSPIRE].

[31] R.H. Horn and C.R. Johnson, Matrix analysis, second ed., Cambridge University Press, New York U.S.A. (2013).

[32] K. Steinhauer and U. Wenger, Loop formulation of the supersymmetric nonlinear $O(N)$ $\sigma$-model, PoS (LATTICE 2013) 092 [arXiv:1311.5403] [INSPIRE].

[33] K. Steinhauer, Loop formulation in low dimensional supersymmetric models on the lattice, Ph.D. thesis, Institute for Theoretical Physics, University of Bern, Bern Switzerland (2014).

[34] L.L. Pennisi, Coefficients of the characteristic polynomial, Math. Mag. 60 (1987) 31. 